\title{
El biopoder a prueba de sus formas sensibles
}

\section{Testing biopower through its sensitive forms}

\author{
Michel AgIER \\ École des Hautes Études en Sciences Sociales (Paris) \\ agier@ehess.fr \\ Traducido por Cristina Maidagan y Andrés Davila
}

Recibido: 16.02 .2012

Aprobado definitivamente: 02.10 .2012

\section{RESUMEN}

En este artículo, la propuesta es trabajar dos conceptos foucaultianos desde un punto de vista antropológico: biopoder y heterotopía. Pretende comprender el significado de unos espacios contemporáneos, tales como la prisión, el campo o el ghetto, mostrando sus relaciones y sus especificidades. El objetivo es establecer un diálogo entre la antropología y la filosofía, elaborando conceptos desde el trabajo de campo.

Palabras Clave: biopoder, heterotopía, migración

\begin{abstract}
In this text, the proposal is to work with two foucauldian concepts from the anthropological point of view: biopower and heterotopy. It aims to understand the meaning of contemporary spaces as prison, camp and ghetto, showing their relations and their specificities. The proposal is also to establish a dialogue between anthropology and philosophy, elaborating concepts from the field.
\end{abstract}

KEYwORDs: biopower, heterotopy, migration

\section{SUMARIO}

1.Biopoder, campo y política: una filosofía sin sujeto. 2.Acerca de los trozos de espacios flotantes. Del biopoder a las heterotopías. 3. Prisión, asilo, refugio. Figuras contemporáneas de la heterotopía 
En este texto propongo trabajar, desde el punto de vista antropológico, mediante conceptos provenientes del análisis foucaultiano, en concreto los de biopoder y heterotopia; éstos, sin llegar a superponerse, "van de la mano» y se interpelan, como se verá aquí a partir de la experiencia habida por los individuos que son su objetivo u objeto. Para el antropólogo se trata de construir una problemática de las emergencias y de las génesis (más que de las pérdidas) de localidades e identidades, y yendo más allá también de comprender el exilio como condición individual y política mayor del siglo XXI, en lugar de contentarse con denunciar el drama del desenraizamiento. En una perspectiva más amplia, la antropología de lo contemporáneo se propone pensar el presente y el porvenir a partir de situaciones vividas y observables hoy en día, de una «etnología del presente» (en palabras de Gérard Althabe) y en la «contemporaneidad» misma del investigador en relación a sus objetos de investigación (como bien lo han mostrado tanto Johannes Fabian como Marc Augé), en vez de partir de reconstrucciones de identidades, territorios, memorias y otras retóricas inspiradas en que esta contemporaneidad es prolija, lo que ha podido (y todavía puede) tanto fascinar como cegar a una etnología chapada a la antigua. Pero se trata asimismo de buscar, en esta perspectiva de reconstrucción y actualización de la antropología, un terreno de intercambio, de diálogo entre antropología y filosofía.

\section{BIOPODER, CAMPO Y POLÍTICA: UNA FILOSOFÍA SIN SUJETO}

El «biopoder» es un poder de vida, y de muerte; si tuviera un horizonte político, éste sería el totalitarismo, esto es, la tanatopolítica, en el sentido de que, para decirlo a la manera de Hannah Arendt, «el totalitarismo no tiende hacia un reino despótico sobre los hombres, sino hacia un sistema en el que los hombres están de más» (Arendt, 1995: 197; Caloz-Tschopp, 2000). En este equilibrio inestable entre la vida y la muerte, el biopoder designa una «tecnología del poder» que supone una transparencia entre el individuo y la «población». Un conjunto de instrumentos se ocupan, controlan y gestionan la vida de las «poblaciones»: de las vidas silenciosas cuando son reducidas a la ficción minimalista de una masa de individuos socialmente excluidos y devueltos al cuerpo común de «la especie humana»; incluso a animales a tenor de la actual asunción de los efectos del absoluto «derecho a la vida» (Foucault, 1997; Brossat, 2010). Estas poblaciones de la especie humana desencarnada y sin alma (o «vidas desnudas», que comentará después Agamben) devienen manejables al ser objetivadas y categorizadas por la medida, la cuenta, el filtro, eliminando por tanto la subjetividad del relato político o social de cada una de esas vidas. Se da así una oposición o tensión bastante lógica y clara, estructuradora desde el punto de vista de la utilización analítica de conceptos, entre el biopoder y la política, sea cual fuere la finalidad del biopoder (salvar vidas, dejar morir a otros, mantener ciertas «poblaciones» al margen, para protegerlas o para protegernos de ellas, etc.).

Pero ahí donde Foucault vinculaba biopoder y saber (e incluso saber y tecnología), Giorgio Agamben (1995 y 1997) ha fusionado biopoder y política. Proponiendo un nivel de abstracción y de ampliación política para el concepto inspirado en los análisis de Foucault pero llevándolo hasta un punto donde éstos no habían llegado, Agamben hace del biopoder el horizonte final de la política en el mundo contemporáneo. Y lo hace antes que nada otorgándole un lugar ejemplar a la forma del «campo».

La transformación del biopoder en un modelo situado en el corazón de la política, asociada a la idea de que la encarnación exacta del mismo sería el campo: la formulación es circular, pesimista y retórica, rizando el rizo, y sin embargo tiene la ventaja de relanzar la investigación empírica, ja modo de un sobresalto! Pues mediante esta figuración abstracta y deductiva, el campo es en definitiva reducido a un puro espacio de muerte, como lo han sido los campos de exterminio nazis a los que Agamben reduce finalmente la figura y el sentido del campo en general, frecuentados por el «espectro del genocidio», inscritos en el único «horizonte de la muerte», como lo ha señalado Alain Brossat (2008). Este enfoque prohíbe ver que, por una parte, el genocidio no necesita en absoluto del campo -ya que puede darse en la calle, como en Kigali durante 1994-, y por otra parte que los campos representan espacios multiformes y multifuncionales. Espacios del gobierno mundializado de los indeseables, pero 
también espacios de socialización en la excepción ordinaria, y espacios políticos a fin de cuentas (Agier, 2008; Bernardot, 2008; Kobelinski y Makaremi, 2009).

Además, a tenor del punto de vista de la teoría antropológica y de los debates que la animan, la superposición entre biopoder y política ya no se sostiene. De manera más precisa, la fórmula según la cual «el campo es el paradigma mismo del espacio político en el momento en que la política deviene biopolítica» (Agamben, 1997: 184) supondría, para ser exacto, un funcionamiento estructural de los espacios sociales, un mundo sin sujetos, lo que permitiría una reificación pura y dura del biopoder. Toda esta representación deja de lado la cuestión de los sujetos y de la subjetivación política ${ }^{1}$. Y esta misma ausencia permite concluir lógicamente el «fin de la ciudad» y de la dominación del campo como «paradigma biopolítico de Occidente». De este modo se confunde la política con el ejercicio del biopoder cuya especialización corre a cargo del campo, mientras la cuestión de la subjetivación permanece inexplorada.

\section{ACERCA DE LOS TROZOS DE ESPACIOS FLOTANTES. DEL BIOPODER A LAS HETEROTOPIAS.}

Esta relación a tres bandas -biopoder, política y campo- es lo que quisiera contestar aquí, para oponerle finalmente las formas del campamento y del gueto, a la vez, como formas sensibles del biopoder y como lugares de una subjetivación que eventualmente escapa al orden del biopoder y le contradice e incluso se le opone a través de la política. En efecto, la cuestión que el antropólogo puede oponer a esta visión sin sujeto de un poder sobre la vida, que sin embargo permanece apremiante y dominante, es antes que nada la de si ¿se puede verificar el biopoder y cuál es su alcance? Conviene retomar la realidad gubernamental que el mismo instituye antes de pensarlo en el corazón y en el principio de la política. Antes de verificar su «verdad», hay la realidad que construye a través de artefactos y de espacializaciones, es decir a través de indispensables formas: será preciso trazar el perímetro para poder recorrer la superficie, y hacer la etnografía. De ahí el vínculo lógico, inmediato desde el punto de vista de la investigación, entre el biopoder y las heterotopias, en lugar del vínculo abstracto entre biopoder y política.

Me baso aquí en un material procedente de estudios en campos de refugiados, de desplazados internos, así como en campamentos «autoestablecidos» principalmente en África negra y de manera más sucinta tanto en el Próximo Oriente como en Europa (Agier, 2008; Agier y Prestianni, 2010). De estos lugares de estudio, todos ellos establecimientos humanos separados del orden social y estatal tan visible como accesible, se puede decir que son «productos derivados» de las verificaciones sociológicas del biopoder -en tanto que gestión de las poblaciones y de los territorios, bajo la forma de un gobierno de los indeseables-, y que son heterotópicos, es decir «un tipo de lugares que están fuera de todo lugar, a pesar de que sean efectivamente localizables» (Foucault, 1984). Que resulten localizables nos permite precisamente observarlos, pasar una temporada en ellos, e intentar comprender in situ su experiencia interior para describirlos. Tales «lugares fuera de todo lugar» -a los que me he referido como fuera-de-lugar de manera genérica en mis investigaciones sobre los campos, campamentos y guetos-, ponen en práctica tres principios de exterioridad -tres ex que encarnan, en última instancia, la potencia de rechazo detentada por un poder soberano: la extraterritorialidad, la

${ }^{1}$ Una antropología del sujeto supone una reflexión que obviamente desborda el propósito de este artículo, pero permite comprender y extraer las consecuencias de la profunda divergencia entre, por una parte une «subjetivación» en el sentido foucaultiano que nos reenvía de hecho a la sujeción, es decir al estar sujetos tanto de los cuerpos como de las categorías identitarias a un poder soberano, en una perspectiva que se mantiene predominantemente estructuralista, y por otra parte una subjetivación en el sentido del sujeto de habla, según un análisis situacional, del momento y movimiento de emancipación cuyo sentido político se construye contra la asignación identitaria y contra el orden del biopoder, y en el límite como una anti-estructura. Que esta anti-estructura adopte a veces el lenguaje de la comunidad contra la sociedad, no tiene nada de sorprendente, e incluso es una figura hasta clásica en antropología de iniciativa de revuelta y rechazo. El sentido de la actuación del sujeto difiere totalmente a partir de esta bifurcación entre estructura y situación. 
excepción, la exclusión. Recorriendo las heterotopías se entra por tanto en la realidad del biopoder.

Las heterotopias se constituyen antes que nada como afueras, situadas en los bordes o los límites del orden normal de las cosas; y son así caracterizadas por el confinamiento y por la extraterritorialidad. Otra noción foucaultiana, aquella de los «encerrados afuera», incluso si ésta ha permanecido en el estado de eslogan, acude en apoyo de la descripción de las heterotopias: es a propósito de la «boat people» de Vietnam, en los barcos atestados de refugiados a la deriva, que a comienzos de los años 1980 Michel Foucault declara, en una intervención militante: «iLos refugiados son los primeros encerrados afuera!». Él mismo había dicho algunos años antes: «El navío, es la heterotopia por excelencia» ${ }^{2}$. Un inventario de los «trozos de espacio flotantes» (Foucault, 2009: 35) es posible: por mi parte, lo detallo y analizo más adelante bajo la representación de un esquema de formas y figuras ligadas entre sí. Deberían incluirse también las pequeñas islas de Nauru y de Christmas en el océano Pacífico, que son utilizadas por el gobierno australiano como vastos centros de retención para exiliados afganos y srilankeses a quienes se les coloca así en posición de incapacidad para penetrar en territorio australiano al objeto de solicitar asilo, pero también deberían incluirse a los navíos demandados en Europa por un alto funcionario griego en el Mediterráneo al objeto de detener, retener y enviar de vuelta a migrantes antes de que pongan un pie sobre suelo europeo. Barcos, islas, zones de attente ${ }^{3}$ portuarias, hangares, centros de retención, campos de refugiados: el hecho mismo de que estos fuera-de-lugar estén constituidos de auténticos «trozos de espacio» indica la posibilidad de una aglomeración confinada y duradera, mantenida al margen.

Pero la extraterritorialidad puede devenir asimismo ficción realizada bajo otra forma, como por ejemplo la de zona de espera «flotante»: en el caso de Francia, la llamada ley Sarkozy de noviembre de 2003 definía la extraterritorialidad como todo aquello que rodea al extranjero indeseable: «la zone d'attente se extiende, y sin que para ello se requiera adoptar una decisión particular, a los lugares en que el extranjero debe acudir». Aún más precisa por lo que se refiere a la ficción extra-territorial, la nueva ley relativa «a la inmigración, a la integración y a la nacionalidad», votada en octubre de 2010 por el parlamento francés, crea automáticamente dicha «zone d'attente» en todo lugar, sea cual fuere (una playa, un sendero de montaña...), en el cual un extranjero en «situación irregular» pone el pie en suelo francés a fin de considerarlo jurídicamente fuera del territorio nacional. Allá donde vaya, el cuerpo del extranjero indeseable se rodea de un «lugar» apropiado que se desplaza con él, fuera de todo lugar.

Como puede observarse, un régimen político y jurídico de excepción es asociado a esta extraterritorialidad. En efecto, desde el punto de vista del poder soberano que decreta la heterotopia, la ficción del afuera es un puro espejismo sin pensamiento propio ni identidad: su espacio real está ocupado por el «adentro» de otro Estado, o bien por un artefacto material o jurídico dentro del que excluye. Dicho de otra manera, los «encerrados fuera» son de hecho "puestos al margen dentro». Así, es en una doble constricción entre un adentro inaccesible y un afuera sin sustancia que el biopoder constituye su artefacto -los objetos barco, isla o campo- como lugar de confinamiento y lugar de vida. El mismo parece situarse en medio del vacío pero no obstante se encuentra siempre «pegado» a la frontera de un orden social y nacional. Sin embargo, cualesquiera que sean sus gestores reales (humanitario, administrativo o comunitario), los espacios situados en la heterotopía tienen como característica común alejar, retrasar o suspender cualquier reconocimiento de una equidad política entre los ocupantes de estos espacios otros y los ciudadanos habituales: hay un régimen de excepción

${ }^{2}$ Cf. «Les hétérotopies» (conferencia radiofónica, France Culture, 7-21 diciembre 1966 en Foucault, 2009: 36).

${ }^{3}$ Nos parece que la traducción literal del termino «zone d'attente» por "zona de espera" no daría una idea ni de su origen (reemplazar a las zonas de tránsito), ni de la distinción que en Francia se establece entre detención (con sus zonas) y retención (con sus centros), ni de la excepcionalidad de tales lugares fuera de lugar: lugares de encierro y concentración para quienes son "no admitidos", "solicitantes de asilo", extranjeros en "tránsito interrumpido"... de modo que para preservar su especificidad hemos decidido mantener su denominación original en el texto [N.T.] 
político asociado a estos espacios e implementado por la ficción de la extraterritorialidad. Una puerta, una zanja, una valla o un pórtico deben ser siempre franqueados para integrar esta forma de vida afuera. La relegación puede ser decretada para confinar en algún lugar una "crisis" (los efectos específicos de una enfermedad o de una guerra) o bien una "desviación" (delincuencia social o una situación irregular): es lo que fundamenta, según Foucault (1984: 756), a las clínicas psiquiátricas, prisiones y residencias de ancianos en tanto que heterotopías. Un régimen extrapolítico, fuera de la política, define la gestión de las poblaciones situadas y controladas en el interior de estos espacios, y según asignaciones categoriales exclusivas: personas definidas por una administración como anormales, viejos, refugiados, vulnerables, delincuentes, discapacitados, etc., se reencuentran así identificadas y agrupadas al margen. Pero al fijarlas y reagruparlas en colectivos, esos espacios otros hacen de sus ocupantes parias duraderos, socialmente "otros".

Así, a la excepción en el plano jurídico y político, a la extraterritorialidad en el plano de la organización de espacios, de lugares y de fronteras, se le asocia un tercer término, la exclusión, definida desde el punto de vista de su (no) lugar en la estructura social y estatal. Los primeros espacios donde se va a vivir el refugio auto-establecido son los intersticios, los terrenos baldíos, los bosques (o fragmentos de bosque en las zonas urbanas), los muelles. El estado de abandono de estos espacios confirma e intensifica la ausencia de ciudadanía territorial de quienes los ocupan: ni el Estado del cual tienen su nacionalidad ni el de su exilio les garantizan el ejercicio de una ciudadanía en los lugares liminares donde se encuentran, o solo les garantizan la vida a modo de protección. Y aquí se aviene la retórica humanitaria del "derecho a la vida", en la fina línea que separa el "hacer vivir" del "dejar morir", y que torna aceptable e incluso invisible en democracia, la exclusión que caracteriza a los indeseables de los derechos sociales del ciudadano: "Estamos fuera de la ley, nadie lo sabe, y sin embargo todo el mundo nos trata como tales" (Kafka, 2008: 62). Los campamentos informales y "clandestinos", productos derivados del rechazo, confluyen entonces en esos campos, zonas y centros oficiales, humanitarios y administrativos que son su producto directo. Sus ocupantes son excluidos socialmente, lo que no impide un uso específico y por lo general no oficial de su fuerza de trabajo de manera marginal en algunos sectores con alto consumo de trabajo ocasional y clandestino, caso del pequeño comercio o el trabajo doméstico, la construcción o el trabajo agrícola. La heterotopía no siempre es sinónimo de inactividad económica, y la condición extraterritorial y excepcional existe también para todos los efectos útiles, especialmente para facilitar un uso ilegal de la fuerza de trabajo de tipo estacional, temporal, etc., de éstos y éstas a quienes se les mantiene al margen ${ }^{4}$.

\section{PRISIÓN, ASILO, REFUGIO. FIGURAS CONTEMPORÁNEAS DE LA HETEROTOPÍA}

La instauración del biopoder en las heterotopías constituye un límite cada vez más asentado: un margen urbano, un margen del Estado, un límite de lo humano. Para encontrarlos en el estudio podremos reconocer las heterotopías contemporáneas en las que se asocian los caracteres de extraterritorialidad, excepción y exclusión. A partir de ahí, es posible precisar las cosas y asociar diferentes formas y figuras de aislamiento y de límite. Indirectamente, señalan los terrenos de investigación sobre las heterotopias contemporáneas. Tres figuras de referencia permiten identificarlos: la prisión, el asilo y el refugio. Las tres en estrecha relación las unas con las otras, tanto porque representan una forma de acantonamiento de sus ocupantes (los "encerrados fuera"), como porque se ven caracterizados por la ambivalencia que las une: la gestión de los indeseables se plasma en la población de las cárceles, el encarcelamiento está presente en el asilo, y éste último es para algunos un refugio. Estas figuras están presentes,

${ }^{4} \mathrm{La}$ articulación de los puntos de vista de, por una parte, la gestión de las poblaciones y de los territorios y, por otra, de la gestión y sobre-explotación de la fuerza de trabajo extranjera, aún está pendiente. Acerca de ello consúltense los estudios y las reflexiones que se incluyen en la obra colectiva recientemente organizada por Alain Morice y Swanie Potot (2010). 
personificadas en distintas formas reales de espacios aparte, de espacios otros que se convierten antes o después en los espacios de los otros. La forma de la prisión en un extremo, y la del gueto urbano en otro representan los dos polos límite hacía los que tiende el sentido de estas heterotopias. Es al menos

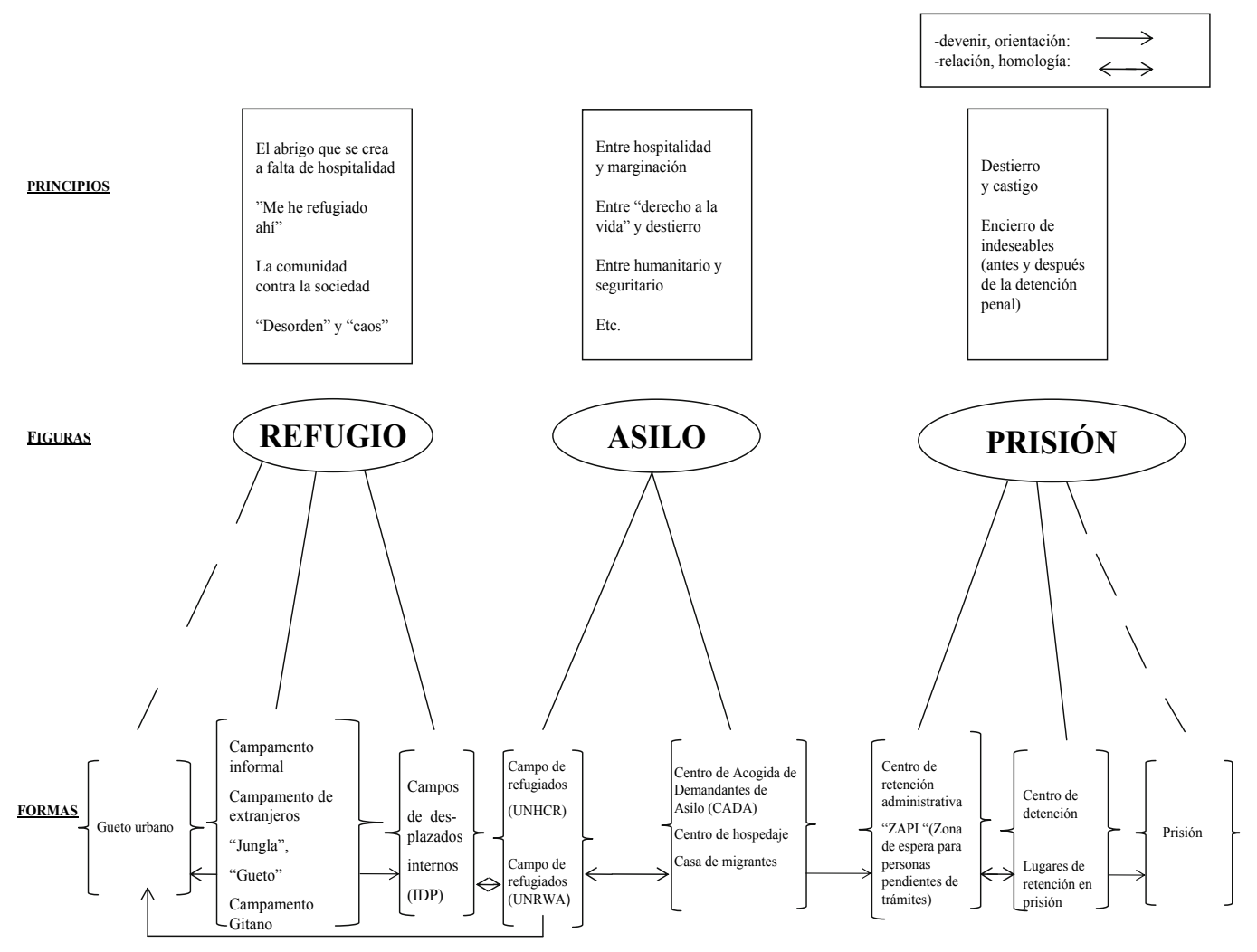

lo que se observa hoy en estos lugares siempre que se hace una etnografía comparativa e integrada. El gráfico adjunto representa este conjunto articulado de figuras y formas.

Algunos comentarios para acompañar dicho esquema. La prisión representa un primer modelo en este paisaje global. Es un polo tanto extremo como ambivalente a la hora de considerar, en general, el sentido y las prácticas del confinamiento de los indeseables. En efecto, la prisión es, en principio, el lugar de un castigo, de un destierro; pero, y especialmente en las prisiones contemporáneas, se ha convertido en espacio de gestión de las poblaciones indeseables: los tiempos de encarcelamiento aumentan, esencialmente antes y después del tiempo de cumplimiento de la pena, es decir, fuera del derecho penal, al igual que la demografía de la población carcelaria, hasta tal punto que la prisión se presenta, hoy en día, como una de las formas de reclusión de los indeseables, especialmente en Francia y Estados Unidos, dos países donde la prisión confina a extranjeros y excluidos ${ }^{5}$. Además, en el marco de la política europea, y en particular en el de la política francesa, de retención y expulsión

${ }^{5}$ Estudios recientes ponen en evidencia el desarrollo de una « prisión para los pobres » en Estados Unidos y de una prisión para los indeseables en Francia donde la cuestión es, cada vez más, la de «encerrar » y de apartar fuera del marco penal propiamente dicho (Wacquant 2004, Combessie 2009). 
son lugares de internamiento administrativos bajo control policial.

La figura del asilo representa un segundo modelo simbólicamente fuerte y asimismo ambivalente: el asilo es acogida y puerta de acceso al mundo común, pero también espacio de reclusión en sí mismo y de relegación de un indeseable (loco, anciano o extranjero). El asilo que se concede lleva el mismo nombre que el asilo que confina. El asilo, en sus muros, encarna una ficción extraterritorial en el seno de las ciudades. Ello remite a los mundos de los Centros de acogida para solicitantes de asilo en Francia (CADA) (Kobelinsky, 2010) y los campos de refugiados del Alto Comisariado de las Naciones Unidas para los Refugiados (ACNUR) de África o Asia. Lo cual explica que los trabajadores sociales que intervienen en las CADA experimenten el mismo malestar que los trabajadores humanitarios de los campos de refugiados. En su vida interna, las formas materiales y sociales sujetas a este principio ambivalente del asilo son lugares de tensión, conflicto o malestar, ya que los protagonistas (acogidos/ encerrados y quienes intervienen en el mismo) son mantenidos, en una temporalidad suspendida, en la incertidumbre de las reglas comunes de su existencia.

Finalmente, la figura del refugio juega un papel en la justificación y gestión de los campos de refugiados pues, como hemos visto, tienen mucho que ver con el asilo de proscritos, la relegación de la territorialidad, de la ley y de la sociedad de los Estados-nación, pero también en lo que se refiere a la forma del campo "autoorganizado". Esto concierne a los campos de IDP (Internally Displaced Persons): lugares de refugio creados por desplazados internos (personas que abandonan su lugar de residencia, pero sin atravesar ninguna frontera nacional). Son campamentos a los que, posteriormente, llegan organizaciones humanitarias, y eventualmente de la órbita de la ONU, con objeto de ayudarles, agruparlos, desplazarlos hacía otro campo, etc. Finalmente, los campos informales de migrantes ("junglas", "guetos", etc.) pueden compararse con las antiguas figuras, generalmente toleradas, de autoinstalación al margen de las ciudades como los campos de extranjeros en África o los campos de los Romà en Europa.

Como sugieren estos breves comentarios, el problema para el análisis foucaultiano es que el espacio otro es, y al mismo tiempo no lo es, el artefacto del biopoder. En otro campo de realidad construida, el de la experiencia sensible y el análisis situacional, es un lugar de socialización emergente, en el límite. Ciertamente, es difícil imaginar el "hacer ciudad" de los campos de refugiados y desplazados, particularmente de África u Oriente Próximo (Agier, 2008), en un centro de retención europeo... pero, por el contrario, es igualmente difícil imaginar la constricción externa (un biopoder que mantiene y tolera fuera de las ciudades a extranjeros que van a establecerse ahí, tal vez, por mucho tiempo) en la definición del campamento autoestablecido y del gueto. Como si el gueto fuera una opción comunitaria intrínseca, interna, que dependiera y llevara a una problemática de la identidad. No sólo la distancia con el Estado (Wacquant, 2006) -y no la etnia, la raza o la religión- define el gueto sino que, más aún, definir su límite es precisamente la expresión del poder gubernamental y por lo tanto de la distancia respecto al Estado. ¿Con arreglo a qué las "ciudades al margen de la ley", según los términos de uno de los líderes del campo afgano de Patras en Grecia, están "fuera" si no es por decisión del Estado? Es el Estado distante el que favorece, o incluso fomenta, la alternativa comunitaria al margen, y así, in fine, organiza tanto como legitima una forma social y política de "guetización" de los espacios de ban-lieu': espacios de "destierro" y del desterrado, mantenidos al margen, en los límites tanto de la ciudad como del Estado. Éste sitúa su propio borde, su "afuera", y en ese fuera, circunscribe y encierra toda idea de una alteridad definida únicamente a través de la disección y la separación, a través de la distancia y por oposición a la Ciudad y al Estado. Así el gueto, más ampliamente el "margen" urbano, no es un borde o un límite más que cuando está al margen del Estado y de los derechos

\footnotetext{
${ }^{6}$ Esto es, « en trámites » $\mathrm{o}$ « pendientes de... » [N.T.]

${ }^{7}$ La palabra ban-lieu no existe como tal. Se trata de un juego de palabras entre banlieue (barrios periféricos ), ban (de bannir: desterrar) y lieu (espacio) [N.T.]
} 
sociales comunes. Ahí también, la extraterritorialidad está muy cerca, por su sentido, de excepción y exclusión.

$*$

Es pues una vida de riesgo, aunque hay que reconocer que aquellos que la viven no se sorprenden de la indiferencia política y del acoso policial al que se enfrentan cada día. Se ríen de ello tanto como lo sufren, lo "utilizan" tanto como lo padecen. El uso de estas autodenominaciones -"gueto", "jungla" "ciudad sin ley"-, utilizadas por los ocupantes de los campos que conocimos sobre el control policial.

La figura del asilo representa un segundo modelo simbólicamente fuerte y asimismo ambivalente: el asilo es acogida y puerta de acceso al mundo común, pero también espacio de reclusión en sí mismo $\mathrm{y}$ de relegación de un indeseable (loco, anciano o extranjero). El asilo que se concede lleva el mismo nombre que el asilo que confina. El asilo, en sus muros, encarna una ficción extraterritorial en el seno de las ciudades. Ello remite a los mundos de los Centros de acogida para solicitantes de asilo en Francia (CADA) (Kobelinsky, 2010) y los campos de refugiados del Alto Comisariado de las Naciones Unidas para los Refugiados (ACNUR) de África o Asia. Lo cual explica que los trabajadores sociales que intervienen en las CADA experimenten el mismo malestar que los trabajadores humanitarios de los campos de refugiados. En su vida interna, las formas materiales y sociales sujetas a este principio ambivalente del asilo son lugares de tensión, conflicto o malestar, ya que los protagonistas (acogidos/ encerrados y quienes intervienen en el mismo) son mantenidos, en una temporalidad suspendida, en la incertidumbre de las reglas comunes de su existencia.

Finalmente, la figura del refugio juega un papel en la justificación y gestión de los campos de refugiados pues, como hemos visto, tienen mucho que ver con el asilo de proscritos, la relegación de la territorialidad, de la ley y de la sociedad de los Estados-nación, pero también en lo que se refiere a la forma del campo "autoorganizado". Esto concierne a los campos de IDP (Internally Displaced Persons): lugares de refugio creados por desplazados internos (personas que abandonan su lugar de residencia, pero sin atravesar ninguna frontera nacional). Son campamentos a los que, posteriormente, llegan organizaciones humanitarias, y eventualmente de la órbita de la ONU, con objeto de ayudarles, agruparlos, desplazarlos hacía otro campo, etc. Finalmente, los campos informales de migrantes ("junglas", "guetos", etc.) pueden compararse con las antiguas figuras, generalmente toleradas, de autoinstalación al margen de las ciudades como los campos de extranjeros en África o los campos de los Romà en Europa.

Como sugieren estos breves comentarios, el problema para el análisis foucaultiano es que el espacio otro es, y al mismo tiempo no lo es, el artefacto del biopoder. En otro campo de realidad construida, el de la experiencia sensible y el análisis situacional, es un lugar de socialización emergente, en el límite. Ciertamente, es difícil imaginar el "hacer ciudad" de los campos de refugiados y desplazados, particularmente de África u Oriente Próximo (Agier, 2008), en un centro de retención europeo... pero, por el contrario, es igualmente difícil imaginar la constricción externa (un biopoder que mantiene y tolera fuera de las ciudades a extranjeros que van a establecerse ahí, tal vez, por mucho tiempo) en la definición del campamento autoestablecido y del gueto. Como si el gueto fuera una opción comunitaria intrínseca, interna, que dependiera y llevara a una problemática de la identidad. No sólo la distancia con el Estado (Wacquant, 2006) -y no la etnia, la raza o la religión- define el gueto sino que, más aún, definir su límite es precisamente la expresión del poder gubernamental y por lo tanto de la distancia respecto al Estado. ¿Con arreglo a qué las "ciudades al margen de la ley", según los términos de uno de los líderes del campo afgano de Patras en Grecia, están "fuera" si no es por decisión del Estado? Es el Estado distante el que favorece, o incluso fomenta, la alternativa comunitaria al margen, y así, in fine, organiza tanto como legitima una forma social y política de "guetización" de los espacios de ban-lieu": espacios de "destierro" y del desterrado, mantenidos al margen, en los límites tanto de la ciudad como del Estado. Éste sitúa su propio borde, su "afuera", y en ese fuera, circunscribe y encierra toda idea de una alteridad definida únicamente a través de la disección y la separación, a través de la distancia y por oposición a la Ciudad y al Estado. Así el gueto, 
más ampliamente el "margen" urbano, no es un borde o un límite más que cuando está al margen del Estado y de los derechos sociales comunes. Ahí también, la extraterritorialidad está muy cerca, por su sentido, de excepción y exclusión.

Es pues una vida de riesgo, aunque hay que reconocer que aquellos que la viven no se sorprenden de la indiferencia política y del acoso policial al que se enfrentan cada día. Se ríen de ello tanto como lo sufren, lo "utilizan" tanto como lo padecen. El uso de estas autodenominaciones -"gueto", "jungla" "ciudad sin ley"-, utilizadas por los ocupantes de los campos que conocimos sobre el terreno con motivo del estudio sobre desplazados, refugiados y migrantes clandestinos debe verse como una forma de objetivación y de afirmación del sujeto. Estos términos dan un sentido propio y asumido (no precisamente "positivo" en el sentido de ser deseables) a los lugares que ocupan y en los que viven.

A semejanza de estas burlas, bromas sobre uno mismo y otras manifestaciones, generalmente ejecutadas de manera dramática, se observa en estos terrenos que la experiencia deconstruye la "realidad" del biopoder. Nos veríamos tentados de decir que simplemente la realidad se "impone" y desplaza el biopoder hacía la ficción, si no se tratara, de hecho y más exactamente, de un conflicto de realidades. Pero la deconstrucción de la realidad que instaura no significa tampoco la no verificación del biopoder. Significa la posibilidad de un actuar político por definición inesperado en estos lugares límite y umbral, en un espacio-tiempo liminar.

\section{BIBLIOGRAFÍA}

Agamben, G. (1995), Moyens sans fins, Notes sur la politique, Rivages.

Agamben, G. (1997), Homo sacer : le pouvoir souverain et la vie nue, Seuil.

Agier, M. (2008), Gérer les indésirables. Des camps de réfugiés au gouvernement humanitaire, Flammarion.

Agier, M. y Prestianni, S. (2010), « Je me suis réfugié là ! ». Bords de routes en exil, ED. Donner lieu.

Arendt, H. (1995), Les Origines du totalitarisme. III - Le système totalitaire, Fayard, col. " Points 》

BeAulieu-GARNiER, E. (2010), Étrangers derrière les barreaux. La prison dans le dispositif de mise à l'écart des étrangers indésirables en France, Memoria de Master (dir: M. Agier), París, EHESS, junio 2010.

Bernardot, M. (2008), Camps d'étrangers, Ed. du Croquant.

Brossat, A. (2008), « L'espace-camp et l'exception furtive », Lignes, n 26, p. 17-19.

Brossat, A. (2010), Droit à la vie ? Seuil.

CAloz-Tschopp, M. (2000), Les sans-État dans la philosophie d'Hannah Arendt, Payot.

Combessie, P. (2009), Sociologie de la prison, La Découverte.

Foucault, M. (1984), « Des espaces autres », en Dits et Écrits, tomo IV, Gallimard, p. 752-762.

Foucault, M. (1997), Il faut défendre la société, Clase en Collège de France, 1975-76, Hautes études, Gallimard/Seuil.

Foucault, M. (2009), Le corps utopique, Les hétérotopies (textos inédito seguidos de una presentación de Daniel Defert), Nouvelles éditions Lignes.

Genel, K. (2004), « Le biopouvoir chez Foucault et Agamben.», Methodos, 4, http://methodos.revues. org/131

KafKA, F. (2008), Journal intime (1945), París, Payot.

Kobelinsky, C. (2010), L'accueil des demandeurs d'asile: Une ethnographie de l'attente, Ed. du cygne.

Kobelinsky, C. y Makaremi, Ch. DiR. (2008), Enfermés dehors. Enquêtes sur le confinement des étrangers, Ed. Le Croquant.

Morice, A. y Ротот, S. DIR. (2010), De l'ouvrier immigré au travailleur sans papiers. Les étrangers dans la modernisation du salariat, Karthala.

Muhle, M. (2006), « Le camp et la notion de vie », in O. Le Cour Grandmaison, G. Lhuilier et J. Valluy (dir.), Le Retour des camps ? Sangatte, Lampedusa, Guantanamo ..., París, Autrement, p. 68-76.

WaCQuant. L. (2004), Punir les pauvres, Agone.

Wacquant. L. (2006), Parias urbains. Ghetto, banlieues, État, La Découverte. 